\title{
Relationship between change in in-vivo exposure distress and PTSD symptoms during exposure therapy for active duty soldiers
}

\author{
Aaron M. Norr ${ }^{\mathrm{a}, \mathrm{b}}$, Kyle J. Bourassa ${ }^{\mathrm{c}, \mathrm{d}}$, Elizabeth S. Stevens ${ }^{\mathrm{c}, \mathrm{e}}$, Matthew J. Hawrilenko ${ }^{\mathrm{c}, \mathrm{b}}$, \\ Scott T. Michael ${ }^{\mathrm{c}, \mathrm{b}}$, \& Greg M. Reger ${ }^{\mathrm{c}, \mathrm{b}}$ \\ ${ }^{a}$ VISN 20 Northwest Network Mental Illness Research, Education and Clinical Center \\ (MIRECC) \\ ${ }^{\mathrm{b}}$ University of Washington School of Medicine \\ ${ }^{c}$ VA Puget Sound Health Care System \\ ${ }^{\mathrm{d}}$ University of Arizona \\ ${ }^{\mathrm{e}}$ University of Illinois at Chicago
}

Author Note

This research (secondary data analysis of previously collected data) was supported by the U.S. Army Medical Research and Materiel Command Military Operational Medicine Research Program [grant number W81XWH-08-2-0015]. The views, opinions, and/or findings contained in this document are those of the authors' and should not be construed as an official Department of Defense or Department of Veteran Affairs position, policy or decision unless so designated by other official documentation. Role of the sponsors: The sponsors had no role in the design, analysis, interpretation, or publication of this study. Declarations of interest: None.

Correspondence concerning this article should be addressed to Greg M. Reger, VA Puget Sound - American Lake Division, 9600 Veterans Drive, A-116

Tacoma, WA 98493. Email: Greg.Reger@va.gov 


\begin{abstract}
Objective: The current study sought to examine the relationship between changes in distress for items on in-vivo exposure hierarchies and posttraumatic stress disorder (PTSD) symptom change over the course of exposure therapy. Methods: Active duty army soldiers $(N=108)$ were recruited from a military base in the U.S. and were enrolled in a randomized clinical trial comparing Prolonged Exposure (PE), Virtual Reality Exposure (VRE), and a wait-list control for the treatment of PTSD stemming from deployments to Iraq or Afghanistan. PTSD diagnosis followed DSM-IV-TR criteria. Outcome measures were assessed via self-report and clinician interview. The relationships between in-vivo exposure distress, imaginal exposure distress, and PTSD symptoms, were examined in a factor of curves model for participants in the treatment conditions. Results: Analyses revealed that, when controlling for one another, changes in in-vivo exposure distress were significantly associated with changes in PTSD symptoms $(\beta=0.75,95 \%$ CI $[0.60,0.90])$, while changes in imaginal exposure distress were not $(\beta=0.03,95 \%$ CI $[-0.27$, 0.33]). The model also revealed that after accounting for the shared variation in trajectories of change, symptom clusters did not have unique variation, meaning that symptom clusters did not change independently. Conclusion: Results suggest the possibility that in-vivo exposures are more closely tied to changes in overall PTSD symptoms than imaginal exposures during exposure therapy. Furture research should incorporate more frequent measurement of in-vivo exposure distress to better elucidate these relations over the course of treatment.
\end{abstract}

Keywords: prolonged exposure, virtual reality exposure, PTSD, in-vivo exposure, imaginal exposure 


\section{Relationship between change in in-vivo exposure distress and PTSD symptoms during exposure therapy for active duty soldiers}

Posttraumatic stress disorder (PTSD) is highly prevalent, with recent lifetime estimates around $8 \%$ of the U.S. population (Kessler, Petukhova, Sampson, Zaslavsky, and Wittchen, 2012). PTSD prevalence among U.S. military personnel and veterans is even higher, with $13 \%$ of those deployed to Iraq or Afghanistan meeting criteria for PTSD (Hoge, Riviere, Wilk, Herrell, and Weathers, 2014). Within a learning theory framework, PTSD has been conceptualized as a disorder that arises in part due to a failure of the natural extinction process following trauma exposure (Rothbaum and Davis, 2003). This conceptualization has been supported by research demonstrating that the vast majority of individuals exposed to a traumatic event experience symptoms of PTSD (i.e., re-experiencing, avoidance, hyperarousal) immediately following the trauma, and natural recovery occurs for many over the subsequent weeks and months (Rothbaum, Foa, Riggs, Murdock, and Walsh, 1992).

According to conditioning theory, the traumatic event itself serves as an unconditioned stimulus (US) which elicits an unconditioned response (UR; e.g., fear, horror). Other stimuli present at the time of the event become conditioned stimuli (CSs), which when subsequently experienced outside the context of the traumatic event reliably elicit a conditioned response (CR). Through the processes of stimulus generalization and higher order conditioning additional previously neutral stimuli in the individual's internal (e.g., intrusive images) and external environment (e.g., situations that were neutral at the time of trauma) become CSs, and elicit a CR (Rothbaum and Davis, 2003).

Exposure therapy is a cognitive behavioral treatment for PTSD designed to facilitate the extinction process (Foa, Hembree, and Rothbaum, 2007). There are various exposure therapy 
protocols in the literature (e.g., Beidel, Frueh, Neer, and Lejuez, 2017; Foa et al., 2007; Keane, Fairbank, Caddell, and Zimering, 1989), but most include some components of repeated imaginal exposure to the trauma memory in-session (imaginal exposure), and repeated in-vivo exposure to trauma-related CSs in the individual's environment (in-vivo exposure). Additionally, some protocols have used virtual reality during imaginal exposures (virtual reality exposure; VRE) to simulate the trauma memory in a multi-sensory environment (e.g., Beidel et al., 2017; Reger et al., 2016). Over repeated exposures, new associations are formed between the CSs and the lack of US. This process is thought to facilitate inhibitory learning and reduce distress elicited by the CSs (Craske et al., 2008). Prolonged Exposure (PE; Foa et al., 2007) is the most researched, evidence-based exposure therapy for PTSD. PE has become a gold-standard treatment for PTSD with efficacy among civilians (American Psychological Association, 2017; Foa et al., 2005) and military personnel (Department of Veterans Affairs \& Department of Defense, 2017; Foa et al., 2018; Reger et al., 2016). Indeed, one recent trial found that both PE and PE using virtual reality during imaginal exposures were efficacious in the treatment of PTSD symptoms among active duty military personnel with combat-related PTSD (Reger et al., 2016).

Initially, habituation (i.e., reduction in the distress elicited by the CS) to imaginal and invivo exposure was proposed as a critical mechanism of PE (Foa, Steketee, and Rothbaum, 1989). Distress elicited by the CS is typically measured through patient report using "subjective units of discomfort" (SUDs; Foa et al., 2007). A recent empirical review determined that while there is limited evidence to support within-session habituation as a predictor of PTSD symptom change in PE, most studies report a modest relationship between between-session habituation and clinical outcomes (Cooper, Clifton, and Feeny, 2017). Though research has called into question the mechanistic role of between-session habituation in symptom change (e.g., Bluett, Zoellner, 
and Feeny, 2014; Craske et al., 2008), it is clear that between-session habituation is often at minimum a co-occurring process with symptom change (Cooper et al., 2017).

However, to our knowledge, every study to date examining the relationship between between-session habituation and PTSD symptom change in exposure therapy has investigated this process only for the imaginal exposure component (see Cooper et al., 2017 for a review). Given the theoretical importance of in-vivo exposure to the extinction process of CSs and reduction of PTSD symptoms, it is surprising that in-vivo habituation has not received more attention in the literature. The symptoms of PTSD can be viewed as manifestations of the failed extinction of trauma related external CSs in each symptom cluster. For example, re-experiencing symptoms include intense psychological distress and physiological reactivity on exposure to external cues that symbolize or resemble the trauma event, whereas the avoidance cluster includes efforts to avoid activities, places, or people that arouse recollections of the trauma. Additionally, it is quite possible that negative alterations in cognition, particularly a continuing belief that the world is a dangerous place, maintains the strength of the CR since it may lead to avoidance of new learning that would reduce the impact of the connection between CS and CR, which was learned at the time of trauma. Finally, symptoms of increased arousal are often seen in response to exposure to trauma-related CSs. Accordingly, it remains an important question whether changes in distress elicited by external CSs over the course of treatment is a predictor of overall PTSD symptom change, as well as change in specific PTSD symptom clusters.

The purpose of the current study is to examine the relationship between changes in invivo SUDs and PTSD symptom change over the course of PE and VRE in a sample of active duty soldiers with combat-related PTSD. Prior published work from this trial demonstrated that PE and VRE significantly reduced PTSD symptoms compared to a waitlist control condition, and 
found no significant differences between the PE and VRE conditions at post-treatment (Reger et al., 2016). It was also established in this sample that changes in SUDs recorded during imaginal exposure were associated with changes in PTSD symptoms (Reger et al., 2019). We hypothesized that changes in in-vivo SUDs would be positively associated with PTSD symptom change over the course of treatment. Second, we proposed that changes in in-vivo SUDs would be uniquely associated with change in each PTSD symptom cluster. Finally, it was hypothesized that changes in in-vivo SUDs and changes in imaginal SUDs would each predict unique variance in PTSD symptom change, as they reflect parallel yet distinct therapy targets (Foa et al., 2007).

\section{Materials and Methods}

\section{Participants}

Participants were drawn from 162 active duty U.S. military personnel with prior combat deployments to Iraq or Afghanistan who enrolled in a randomized controlled trial that compared the efficacy of PE or VRE to a waitlist control (see Reger et al., 2016). This secondary analysis included those who were randomized to receive active treatment $(n=108)$, which included 10 sessions of PE or VRE. Participants were predominantly male $(95 \%, n=103)$, Caucasian $(64 \%$, $n=69)$, and had some college education $(67 \%, n=72)$. Full demographics are available elsewhere (Reger et al., 2016).

Participants were included if they met full $D S M-I V$-TR criteria for PTSD related to combat trauma experienced during deployment, as assessed by the Clinician-Administered PTSD Scale (CAPS; Blake et al., 1995). The index trauma was required to have occurred at least three months before the baseline assessment and in a setting that could be simulated by the Virtual Reality Iraq/Afghanistan software (Rizzo, Reger, Gahm, Difede, and Rothbaum, 2009). Exclusion criteria were: (1) changes in psychotropic medications in the last month; (2) 
hospitalization for risk of suicide or self-harm in the last six months; (3) ongoing threats to safety (e.g., domestic violence); (4) current alcohol or drug dependence; a history of (5) bipolar or psychotic disorder, (6) seizures, or (7) loss of consciousness for more than 15 minutes since entering military service; (8) physical conditions that would preclude engagement with the VRE system; (9) prior treatment with PE; (10) concurrent other psychotherapy for PTSD. Participants were asked to not initiate new psychotropic medications or therapies during their participation in the trial.

\section{Procedure and Treatments}

After providing informed consent, participants completed baseline assessment of inclusion and exclusion criteria, demographics, self-report surveys, the baseline CAPS assessment, and a physiological assessment. Assessment time points included a mid-treatment assessment after the $5^{\text {th }}$ session of therapy and a post-treatment assessment after session 10 . On average, participants completed the mid-treatment assessment 31.2 days $(S D=19.5)$ after initiating treatment, and completed the post-treatment assessment 70.9 days $(S D=36.97)$ after initiating treatment. Three-month and 6-month follow-up assessments were also conducted but not used for the purposes of this study.

Therapy was conducted according to the published PE treatment manual (Foa et al., 2007) and consisted of 10 approximately weekly sessions, each 90-120 minutes in length. The following is a description of the PE treatment protocol. Session 1 included psychoeducation on exposure therapy, assessment of trauma history and its impact, and introduction of breathing retraining. In Session 2, clinicians collaboratively discussed common reactions to trauma, introduced in-vivo exposure, and developed SUDs anchor points. During this session, clinicians also assisted the client in constructing an in-vivo exposure hierarchy by helping them generate a 
list of situations, places, or circumstances they avoided. Participants rated their SUDs for how distressed they would feel participating in each item on the list. In-vivo exposures were assigned as daily homework from Session 2 onward. Specifically, clients were asked to intentionally approach safe, but anxiety-provoking situations on their in-vivo hierarchy, and to remain in the situation until distress decreased. In Session 3, the rationale and procedures for imaginal exposure were introduced. During imaginal exposure, clients repeatedly, verbally recounted the trauma, progressively incorporating sensory and emotional detail, for 30-45 minutes per session. Clinicians elicited clients' SUDs ratings every five minutes. Afterward, clinicians processed the imaginal exposure experience. Imaginal exposures continued in sessions 4-10. In Session 10, participants completed a final imaginal exposure and re-rated each in-vivo exposure hierarchy item with their current SUDs for each item on the list.

Participants assigned to the VRE condition received identical treatment except that imaginal exposures were conducted using virtual reality. Participants wore a head-mounted display and high fidelity over-the-ear headphones while seated or standing on a platform that vibrated during low-frequency sounds. While clients recounted the memory aloud, the clinician used the virtual reality interface to simultaneously alter the virtual reality environment or stimuli to match the narrative. There were no differences in how in-vivo exposure was delivered for both conditions. For more detail on this system, see Rizzo and colleagues (2009). Given previous findings that there were no differences between PE and VRE at post-treatment (Reger et al., 2016), the two active treatment groups were combined for the purposes of this study.

\section{Measures}

Clinician-Administered PTSD Scale (CAPS; Blake et al., 1995). The CAPS is a structured diagnostic interview that assesses the presence and severity of symptoms of PTSD 
according to DSM-IV-TR criteria. Psychologists coded the frequency and intensity of each symptom on a 0 (Never; None) to 4 (daily or almost every day; extreme distress) scale. For this paper's analyses, we used a "last week" time reference to support detection of symptom changes between assessment points. In this subsample, internal consistency of the CAPS was good at baseline $(\alpha=0.81)$ and was excellent at mid-treatment $(\alpha=0.94)$ and post-treatment $(\alpha=0.96)$.

Subjective Units of Discomfort (SUDs; Foa et al., 2007). SUDs is a self-reported index of the intensity of anxiety, discomfort, or distress experienced in a specified moment. SUDs was rated on a continuous $0-100$ scale, where 0 represents no distress and 100 represents extreme (maximum) distress. Participants collaborated with their therapist to "anchor" SUDs ratings to idiographic past experiences, and then provided SUDs ratings throughout exposure therapy to indicate their state-dependent levels of distress. We distinguish between in-vivo SUDs (defined as the SUDs ratings that participants assigned to each item on the in-vivo exposure hierarchy) and imaginal SUDs (defined as average SUDs during that session's imaginal exposure). Consistent with the PE treatment protocol (Foa et al., 2007), initial in-vivo SUDs were collected at Session 2 during hierarchy construction, and initial imaginal SUDs were collected during the first imaginal exposure (average of all ratings during session 3) in the third session of treatment. Final in-vivo SUDs and final imaginal SUDs (average of all ratings during session 10) were collected at last session of treatment. In-vivo SUDs ratings from the exposures themselves were not collected by study staff in this trial and therefore were not available for the current analyses.

\section{Data Analysis}

To differentiate the shared and unique aspects of changes in PTSD symptoms, we used a factor of curves model (FOC; Isiordia, Conger, Robins, and Ferrer, 2017; McArdle, 1988). The FOC model is a second-order factor model that captures shared variation in trajectories of change 
across symptom clusters. Latent growth models (Cole and Maxwell, 2003) were fit to each symptom cluster. Two second-order factors, one measured by the intercepts and one measured by the slopes of the growth factors, were used to capture the change that was shared across symptom clusters (i.e., PTSD symptoms). Under this parameterization, the change shared by all symptoms was captured by the second-order slope term, and cluster-unique aspects of change were captured by the residual variances of the first-order slope terms. A statistically significant residual would indicate that a symptom cluster had variability not explained by the general pattern of change.

We planned to test whether in-vivo SUDs were associated with unique changes in symptom clusters by comparing alternative models where changes in in-vivo SUDs scores predicted the common slope factor (second-order term) versus a model where they predicted the 3 unique symptom cluster slopes (first-order slope terms). A significant chi-square difference test between models would indicate that the higher order model was a worse fit to the data. To test whether in-vivo and imaginal SUDs predicted unique variance in PTSD symptom change, we included change in average imaginal (average session 10 - average session 3) SUDs scores across treatment as an additional predictor in the model determined in the previous step. We included age, gender, and initial SUDs scores of each type as covariates in our models. To account for missing data in all models, we used full likelihood maximum likelihood (FIML) in MPLUS version 7.31 (Muthén and Muthén, 1998-2012). FIML produces unbiased estimates under conditions of missing at random (i.e., missingness is adequately explained by variables included in the model). FIML outperforms other missing data treatments, such as listwise deletion and similar response pattern imputation (Graham, 2009).

\section{Results}




\section{Preliminary Model Specification}

To ensure good local fit before building the second-order model, latent growth models were fit to each of the three PTSD symptom clusters independently. Both hyperarousal and avoidance/numbing symptoms were well represented by linear change. Reexperiencing symptoms had curvilinear change, which we modeled with a quadratic term with the variance fixed to zero. Consistent with prior work on this sample (Reger et al., 2016), all symptom clusters decreased markedly over time (reexperiencing Cohen's $d=-0.74$, hyperarousal $d=$ 1.44 , avoidance/numbing $d=-1.68$, calculated using the mean change over the study divided by the standard deviation of the cluster score at baseline for each independent growth model). Fit for these preliminary models can be found in Table 1 .

\section{Specifying the LCGM for PTSD Symptoms}

To understand shared versus unique variability in symptom change, the FOC model was fit to the first-order cluster terms. This model adequately fit the data, $\chi^{2}(25, N=108)=43.04, p$ $=.014, \mathrm{CFI}=0.95, \mathrm{RMSEA}=.08,90 \% \mathrm{CI}[.04, .12]$, though the significant $\chi^{2}$ suggests some model misfit. Notably, chi-square tests can be overly conservative when sample sizes are less than 200 (McNeish, 2018). In order to test whether cluster scores had unique change over and above that accounted for by the common slope factor, we then tested whether constraining the residual variances for the three clusters to 0 reduced model fit. Doing so did not reduce fit, $\Delta \chi^{2}$ $(4, N=108)=4.34, p=.36$, providing evidence that the second-order factor explained the relevant variance in PTSD symptoms change. This suggests there is not significant variation in the trajectory of PTSD symptom clusters during treatment, and therefore we only predicted variation in the second-order overall PTSD symptom factor in our subsequent models.

\section{Changes in In-Vivo SUDs Predicting PTSD Symptoms}


We next tested whether initial levels or changes in self-reported in-vivo SUDs predicted the intercept and slopes of PTSD symptoms. We regressed the intercept of PTSD symptoms on the initial level of in-vivo SUDs, and regressed the slope of PTSD symptoms on both initial level and changes in in-vivo SUDs. Initial in-vivo SUDs at session 2 predicted the intercept of PTSD symptoms, $\beta=0.35,95 \%$ CI $[0.08,0.62], p=.010$. Change in in-vivo SUDs from session 2 to session 10 significantly predicted the slope of PTSD symptoms, $\beta=0.77,95 \%$ CI $[0.65,0.89], p$ $<.001$, whereas initial in-vivo SUDs at session 2 did not, $\beta=0.03,95 \% \mathrm{CI}[-0.16,0.22], p=$ .786 .

\section{Changes in In-Vivo and Imaginal SUDs Predicting PTSD Symptoms}

We next compared the association of initial level and changes in in-vivo SUDs when also including initial level and changes in imaginal self-reported SUDs ${ }^{1}$ predicting the intercept and slope of PTSD symptoms. We regressed the intercept of PTSD symptoms on the initial level of in-vivo and imaginal SUDs, and regressed the slope of PTSD symptoms on both initial level and changes in both in-vivo and imaginal SUDs. Initial in-vivo SUDs at week 2 remained significantly associated with the intercept of PTSD symptoms, $\beta=0.26,95 \% \mathrm{CI}[0.01,0.51], p=$ .044. Initial imaginal SUDs at session 3 was independently significantly associated with the intercept of PTSD symptoms, $\beta=0.35,95 \% \mathrm{CI}[0.10,0.60], p=.006$. Change in in-vivo SUDs remained significantly associated with change in PTSD symptoms, $\beta=0.75,95 \%$ CI $[0.60$, 0.90], $p<.001$, but changes in imaginal SUDs did not predict change in PTSD symptoms after controlling for changes in in vivo SUDS, $\beta=0.03,95 \%$ CI $[-0.27,0.33], p=.859$. Neither initial in-vivo SUDs nor initial imaginal mean SUDs predicted change in PTSD symptoms. Initial in-

\footnotetext{
${ }^{1}$ All models that included change in mean imaginal SUDs and initial mean SUDs were also tested using change in and initial peak SUDs. Using peak imaginal SUDs did not alter any of the substantive results of the current study.
} 
vivo and imaginal SUDs, $r=0.27, p=.023$, as well as changes in in-vivo and imaginal SUDs, $r$ $=0.60, p<.001$, were significantly correlated.

\section{Discussion}

The current study investigated the relationship between in-vivo distress and PTSD symptoms during exposure therapy among active duty soldiers with combat-related PTSD. Results revealed that decreases in in-vivo SUDs were associated with decreases in PTSD symptoms over the course of treatment. These results are consistent with prior studies which have found relationships between changes in SUDs during imaginal exposure and PTSD symptoms (see Cooper et al., 2017 for an empirical review). However, to our knowledge this is the first study to test a direct, empirical relationship between in-vivo SUDs and PTSD symptoms over the course of PE or VRE. These results are not surprising given the theoretical importance of in-vivo exposures to reduce conditioned responses to generalized conditioned stimuli in the context of PTSD (Rothbaum and Davis, 2003), and further suggest that between session habituation (for both imaginal and in-vivo exposures) is often at minimum a co-occurring process with symptom change within exposure therapy for PTSD, if not a mechanistic process, per se (Cooper et al., 2017).

Though prior research in this sample found a significant relationship between changes in SUDs during imaginal exposure and changes in PTSD symptoms (Reger et al., 2019), only the relationship between changes in in-vivo SUDs and PTSD symptoms remained significant when both were considered in the same model. These results suggest that changes in in-vivo exposure distress may be more closely related to overall change in symptoms than changes in imaginal exposure distress. These results are logical when considering in-vivo exposures are able to directly target a much broader range of conditioned stimuli than imaginal exposures. Clearly, 
imaginal exposure is a critical component of exposure therapy and effective PTSD treatment. Nonetheless, this study suggests that the reductions in distress elicited by external conditioned stimuli may be more closely associated to overall PTSD symptom change. Therefore, the current results underscore the importance of clinical attention to changes in in-vivo exposure distress. Should future research determine that reductions in in-vivo distress serve as a mechanism of PTSD symptom reduction, clinicians may benefit from monitoring in-vivo distress throughout treatment as an additional indicator of treatment progress. Prolonged exposure is unique among the exposure protocols in that in-vivo exposures are often not directly observed by the clinician, whereas imaginal exposures are. It can be easy, as a PE clinician, to focus on the imaginal exposures, which are more salient in the room, rather than the in-vivo exposures outside, which could lead to less optimal progress for the patient if the patient is not experiencing difficulty with extinction during in-vivo exposures.

Symptom changes for all soldiers followed the same pattern: the largest changes were found in avoidance/numbing, followed by hyperarousal, then re-experiencing, and this ordering was generally consistent across soldiers. Some prior work has found evidence of differences in amounts and rates of change in symptom clusters during group and individual treatment with an exposure component (Bryant et al., 2003; Maples-Keller, Price, Rauch, Gerardi, and Rothbaum, 2017; Phelps et al., 2018; Taylor et al., 2003). Our results were consistent with prior work which found the effect size for re-experiencing symptoms was smaller than the other symptom clusters during exposure therapy (Phelps et al., 2018). However, to our knowledge ours is the first study to directly examine covariation among symptom cluster change over the course of exposure therapy for PTSD. Our findings suggest PTSD symptom clusters changed together rather than independently. 
The current study is not without limitations. First, in-vivo exposure distress was measured based on patients' ratings of exposure situations on their hierarchies during session 2 and session 10, rather than their ratings during the exposures themselves. Measurement of exposure distress during the exposures themselves would be a more precise approach to address the question of habituation across in-vivo exposures and would reduce the possibility of recall bias. It is also possible that patients did not complete all the situations on their hierarchy and some of the ratings are simply their perception of how difficult those situations would be. Future research would benefit from examining this question by assessing real-time SUDs ratings during in-vivo exposure exercises, as has previously been done using SUDs during imaginal exposure. Second, by only measuring in-vivo and imaginal SUDs at two time points, we were unable to assess whether changes in exposure distress preceded changes in PTSD symptoms or vice versa. This is an important area of future research to examine the potential mechanistic role of in-vivo distress change, rather than as a correlate of symptom change. Third, all participants in the current study received both the imaginal (with or without virtual reality assistance) and in-vivo exposure components of exposure therapy and therefore it would be impossible to determine relative importance of these two components as their effects and relations to symptom change are intertwined. It should be noted that one small study ( $N=14$; Richards, Lovell, and Marks, 1994) did find that in-vivo exposure was more effective than imaginal at reducing phobic avoidance (other symptom change was comparable between exposure types), however, those results would clearly need to be replicated in a much larger sample before conclusions could be drawn. Similarly, it would be useful to examine whether or not experiencing a reduction in in-vivo exposure distress, as opposed to not experiencing a reduction in imaginal exposure distress, is associated with poorer treatment outcomes. Finally, given this is the first study to examine the 
relations between in-vivo distress habituation and PTSD symptom change in exposure therapy, it is possible that these results will not generalize to other samples.

Despite these limitations the current study is a significant step forward as it is the first to examine the relationship between in-vivo distress habituation and PTSD symptom change in exposure therapy for PTSD. Results revealed that only the relationship between changes in invivo exposure distress and PTSD symptom change, as opposed to imaginal exposure distress, remained significant when accounting for both in the same model. These results suggest the possibility that in-vivo exposures are more closely tied to changes in overall PTSD symptoms than imaginal exposures. Future research should incorporate more frequent measurement of invivo exposure distress and utilize a dismantling design to better elucidate these relations over the course of treatment and the potential relative efficacy of these treatment components. 


\section{References}

American Psychological Association. 2017 Clinical practice guideline for the treatment of posttraumatic stress disorder (PTSD) in adults [PDF file]. Washington, DC: American psychological association. Retrieved from https://www.apa.org/ptsd-guideline/ptsd.pdf

Beidel, D.C., Frueh, B.C., Neer, S.M., Lejuez, C.W., 2017. The efficacy of trauma management therapy: A controlled pilot investigation of a three-week intensive outpatient program for combat-related PTSD. Journal of anxiety disorders. 50, 23-32.

https://doi.org/10.1016/j.janxdis.2017.05.001

Bluett, E.J., Zoellner, L.A., Feeny, N.C., 2014. Does change in distress matter? Mechanisms of change in prolonged exposure for PTSD. Journal of behavior therapy and experimental psychiatry. 45, 97-104. https://doi.org/10.1016/j.jbtep.2013.09.003

Bryant, R.A., Moulds, M.L., Guthrie, R.M., Dang, S.T., Nixon, R.D.V., 2003. Imaginal exposure alone and imaginal exposure with cognitive restructuring in treatment of posttraumatic stress disorder. Journal of consulting and clinical psychology. 71, 706-712. http://dx.doi.org/10.1037/0022-006X.71.4.706

Cole, D.A., Maxwell, S.E., 2003. Testing mediational models with longitudinal data: Questions and tips in the use of structural equation modeling. Journal of abnormal psychology. 112, 558-577. https://doi.org/10.1037/0021-843X.112.4.558

Cooper, A.A., Clifton, E.G., Feeny, N.C., 2017. An empirical review of potential mediators and mechanisms of prolonged exposure therapy. Clinical psychology review, 56, 106-121. https://doi.org/10.1016/j.cpr.2017.07.003 
Craske, M. G., Kircanski, K., Zelikowsky, M., Mystkowski, J., Chowdhury, N., Baker, A., 2008. Optimizing inhibitory learning during exposure therapy. Behaviour research and therapy. 46, 5-27. https://doi.org/10.1016/j.brat.2007.10.003

Department of Veterans Affairs \& Department of Defense, 2017. VA/DoD Clinical practice guideline for the management of posttraumatic stress disorder and actue stress disorder (Third Edition) [PDF file]. Washington, DC: U.S Department of Veterans Affairs. Retrieved from https://www.healthquality.va.gov/guidelines/mh/ptsd/

Foa, E. B., Hembree, E.A., Cahill, S.P., Rauch, S.A., Riggs, D.S., Feeny, N.C.,Yadin, E., 2005. Randomized trial of prolonged exposure for posttraumatic stress disorder with and without cognitive restructuring: Outcome at academic and community clinics. Journal of consulting and clinical psychology. 73, 953-964. http://dx.doi.org/10.1037/0022006X.73.5.953

Foa, E.B., Hembree, E.A., Rothbaum, B.O., 2007. Prolonged exposure therapy for PTSD: Emotional processing of traumatic experiences. USA: Oxford Universtiy Press.

Foa, E.B., McLean, C.P., Zang,Y., Rosenfield, D., Yadin, E., Yarvis, J.S., . . Peterson, A.L., 2018. Effect of prolonged exposure therapy delivered over 2 weeks vs 8 weeks vs present-centered therapy on PTSD symptom severity in military personnel: A randomized clinical trial. Journal of the american medical association. 319, 354-364. https://doi.org:10.1001/jama.2017.21242

Foa, E.B., Steketee, G., Rothbaum, B.O., 1989. Behavioral/cognitive conceptualizations of posttraumatic stress disorder. Behavior therapy. 20, 155-176. https://doi.org/10.1016/S00057894(89)80067-X 
Graham, J.W., 2009. Missing data analysis: Making it work in the real world. Annual review of psychology. 60, 549-576. https://doi.org/10.1146/annurev.psych.58.110405.085530

Hoge, C.W., Riviere, L.A., Wilk, J.E., Herrell, R.K.,Weathers, F.W., 2014. The prevalence of post-traumatic stress disorder (PTSD) in US combat soldiers: a head-to-head comparison of DSM-5 versus DSM-IV-TR symptom criteria with the PTSD checklist. The lancet psychiatry. 1, 269-277. https://doi.org/10.1016/S2215-0366(14)70235-4

Isiordia, M., Conger, R., Robins, R.W., Ferrer, E., 2017. Using the factor of curves model to evaluate associations among multiple family constructs over time. Journal of family psychology. 31, 1017-1028. https://psycnet.apa.org/doi/10.1037/fam0000379

Keane, T.M., Fairbank, J.A., Caddell, J.M., Zimering, R.T., 1989. Implosive (flooding) therapy reduces symptoms of PTSD in Vietnam combat veterans. Behavior therapy. 20, 245-260. https://doi.org/10.1016/S0005-7894(89)80072-3

Kessler, R.C., Petukhova, M., Sampson, N.A., Zaslavsky, A.M., Wittchen, H.U., 2012. Twelvemonth and lifetime prevalence and lifetime morbid risk of anxiety and mood disorders in the United States. International journal of methods in psychiatric research. 21, 169-184. https://doi.org/10.1002/mpr.1359

Maples-Keller, J.L., Price, M., Rauch, S., Gerardi, M., Rothbaum, B.O., 2017. Investigating relationships between PTSD symptom clusters within virtual reality exposure therapy for OEF/OIF veterans. Behavior therapy. 48, 147-155. https://doi.org/10.1016/j.beth.2016.02.011

McArdle, J.J., 1988. Dynamic but structural equation modeling of repeated measures data. Handbook of multivariate experimental psychology (pp. 561-614): Charlottesville, VA: Springer. 
McNeish, D., in press. Should we use F-Tests for model fit instead of Chi-Square in overidentified structural equation models? Organizational research methods. https://doi.org/10.1177/1094428118809495

Muthén, L.K., \& Muthén, B.O., 1998-2012. Mplus User's Guide: Statistical Analysis with Latent Variables, seventh ed. Los Angeles, CA: Muthén \& Muthén.

Phelps, A.J., Steele, Z., Cowlishaw, S., Metcalf, O., Alkemade, N., Elliott, P., . . Howard, A.J., 2018.Treatment outcomes for military veterans with posttraumatic stress disorder: Response trajectories by symptom cluster. Journal of traumatic stress. 31, 401-409. https://doi.org/10.1002/jts.22299

Reger, G.M., Koenen-Woods, P., Zetocha, K., Smolenski, D.J., Holloway, K.M., Rothbaum, B.O., . . . Skopp, N.A., 2016. Randomized controlled trial of prolonged exposure using imaginal exposure vs. virtual reality exposure in active duty soldiers with deploymentrelated posttraumatic stress disorder (PTSD). Journal of consulting and clinical psychology. 84, 946-959. http://dx.doi.org/10.1037/ccp0000134

Reger, G.M., Smolenski, D., Norr, A., Katz, A., Buck, B., Rothbaum, B.O. 2019. Does virtual reality increase emotional engagement during Exposure for PTSD? Subjective distress during prolonged and virtual reality exposure therapy. Journal of Anxiety Disorders. 61, 75-81. https://doi.org/10.1016/j.janxdis.2018.06.001

Richards, D.A., Lovell, K., Marks, I.M., 1994. Post-traumatic stress disorder: Evaluation of a behavioral treatment program. Journal of traumatic stress. 7, 669-680. https://doi.org/10.1007/BF02103014 
Rothbaum, B.O., Davis, M., 2003. Applying learning principles to the treatment of post-trauma reactions. Annals of the New York Academy of Sciences, 1008, 112-121. https://doi.org/10.1196/annals.1301.012|

Rothbaum, B.O., Foa, E.B., Riggs, D.S., Murdock, T., \& Walsh, W.,1992. A prospective examination of post-traumatic stress disorder in rape victims. Journal of Traumatic stress, 5, 455-475. https://doi.org/10.1002/jts.2490050309|

Taylor, S., Thordarson, D.S., Maxfield, L., Fedoroff, I.C., Lovell, K., Ogrodniczuk, J., 2003. Comparative efficacy, speed, and adverse effects of three PTSD treatments: Exposure therapy, EMDR, and relaxation training. Journal of consulting and clinical psychology, 71, 330-338. https://psycnet.apa.org/doi/10.1037/0022-006X.71.2.330 
Table 1.

Model Fit Change During Model Specification

\begin{tabular}{|c|c|c|c|c|c|}
\hline Change in model specification & $d f$ & $\chi^{2}$ & $p$ & CFI & RMSEA \\
\hline LCGM - Reexperiencing symptom cluster & 0 & & & & \\
\hline LCGM - Avoidance/Numbing symptom cluster & 1 & 0.03 & .874 & 1.00 & .000 \\
\hline LCGM - Hyperarousal symptom cluster & 1 & 0.01 & .938 & 1.00 & .000 \\
\hline Combined model including the symptom cluster LCGM & 17 & 26.74 & .062 & 0.97 & .073 \\
\hline $\begin{array}{l}\text { Combined model including second order PTSD } \\
\text { symptom factor }\end{array}$ & 28 & 47.38 & .013 & 0.95 & .080 \\
\hline Second order factor model including in-vivo SUDs & 57 & 64.97 & .219 & 0.98 & .046 \\
\hline Second order factor model including imaginal SUDs & 72 & 80.36 & .234 & 0.98 & .043 \\
\hline
\end{tabular}

Note: LCGM = Latent curve growth model; CFI = confirmatory fit index; RMSEA = root mean squared error of approximation. 